\title{
Philosophiques
}

\section{Volitions et auto-affection}

\section{Stéphane Chauvier}

Volume 35, numéro 1, printemps 2008

Les valeurs de l'ironie (1) et le scepticisme à l'âge classique (2)

URI : https://id.erudit.org/iderudit/018241ar

DOI : https://doi.org/10.7202/018241ar

Aller au sommaire du numéro

Éditeur(s)

Société de philosophie du Québec

ISSN

0316-2923 (imprimé)

1492-1391 (numérique)

Découvrir la revue

Citer ce document

Chauvier, S. (2008). Volitions et auto-affection. Philosophiques, 35(1), 131-137. https://doi.org/10.7202/018241ar d'utilisation que vous pouvez consulter en ligne.

https://apropos.erudit.org/fr/usagers/politique-dutilisation/ 


\title{
Volitions et auto-affection
}

\author{
STÉPHANE CHAUVIER \\ Université de Caen Basse-Normandie
}

Dans son ouvrage sur la nature de la volonté, Joëlle Proust entend reconsidérer, à la lumière de certains résultats récents de la psychologie et de la neurophysiologie cognitives, le problème philosophique traditionnel de la nature de l'action volontaire. Quelques auteurs, à la suite d'Aristote et de Wittgenstein, soutiennent qu'une action volontaire est simplement un comportement dont celui qui l'adopte peut rendre raison en première personne. D'autres philosophes, à la suite de Descartes et de Locke, tiennent au contraire qu'une action volontaire est un comportement causé par la volonté de l'agent ou, plus exactement, par une volition de l'agent, par une détermination de sa volonté. J. Proust a choisi le second de ces deux partis philosophiques. Elle pense notamment que la conception volitionniste peut permettre d'intégrer, mieux que sa rivale, certains résultats des sciences contemporaines de la cognition et du cerveau. Toutefois, il convient de souligner que le volitionnisme, comme d'ailleurs son contraire, le rationalisme, n'est pas de lui-même une théorie scientifique ou positive. C'est une explication philosophique ou conceptuelle de l'action volontaire permettant, le cas échéant, d'interpréter les résultats des sciences cognitives. L'objet de l'ouvrage de J. Proust est donc non seulement d'interpréter, dans le cadre du volitionnisme, certains développements contemporains des sciences cognitives, mais aussi de donner à la théorie volitionniste une formulation générale qui lui permette de résister aux objections philosophiques ou conceptuelles dont elle a été l'objet.

Dans ses formulations traditionnelles, le volitionnisme se heurte en effet à deux objections conceptuelles principales. En premier lieu à l'objection de la régression infinie: si l'action de lever le bras est causée par la volition de lever le bras et si une volition est conçue comme une "action de l'âme ", cette volition semble appeler une volition antérieure, et ainsi de suite à l'infini (p. 136). En second lieu, cette conception volitionniste semble conduire à la théorie de l'homoncule (p. 175) et, avec elle, à la théorie de l'Ego spirituel: si une volition est une action de l'âme, alors cette action semble appeler un sujet-auteur qui, ne pouvant être le grand agent corporel lui-même, est fatalement un petit agent dans le gros, un Ego spirituel.

J. Proust affirme pouvoir échapper à ce double risque de régression et d'homonculisation, en définissant une volition comme un événement. Un événement, étant impersonnel, ne requiert pas un sujet-auteur. Et un évènement n'étant pas une action, il ne requiert pas non plus une volition antérieure. Une action volontaire est donc simplement l'expansion phénoménale d'une volition. Lorsque nous voyons un homme lever son bras pour attraper une pomme dans un arbre, nous assistons à l'expansion d'une volition dans le monde. 
Quel que soit pour l'instant le détail de cette explication, un point doit d'emblée être souligné. Expliquer philosophiquement un phénomène ne peut évidemment consister à donner une description cohérente d'une partie seulement de ce phénomène. Or considérons à nouveau l'action volontaire consistant à lever le bras pour attraper une pomme dans un arbre. Expliquer ce phénomène requiert évidemment de rendre compte de ce qui fait qu'à un moment donné le bras de l'homme se lève et se dirige vers le fruit. Mais expliquer ce phénomène requiert aussi de rendre compte du bruit caractéristique qui accompagne ou peut accompagner le mouvement de ce bras vers la pomme, si du moins il s'agit d'un bras humain, à savoir l'énoncé: "J'attrape une pomme » que l'agent peut ou pourrait proférer en réponse à une question du genre : "Que fais-tu ? ». Un chimpanzé pourrait attraper cette pomme en silence et la description du mécanisme le conduisant à le faire expliquerait valablement le phénomène. Mais les actions volontaires des hommes sont pénétrées d'énoncés à la première personne ou, au moins, pénétrés de dispositions à en proférer, de sorte que l'explication que l'on propose doit aussi rendre compte de cette pente qu'ont les agents humains à se poser comme les auteurs de leurs actions. La théorie que propose J. Proust y parvient-elle ? C'est sur ce point crucial que nous voudrions nous concentrer.

Pour rendre plus intuitive la discussion, considérons l'exemple précédent, celui d'un homme, Pierre, qui tend son bras pour attraper une pomme dans un arbre. On pourra peut-être trouver ce cas trop simple. Notons toutefois que c'est en songeant à des actions corporelles élémentaires comme celle-ci que J. Proust élabore sa théorie. Ici et là elle affirme que ce qui vaut pour l'action d'attraper un objet ou de glisser un disque dans une fente vaut aussi pour des actions à plus large spectre et à moindre implication corporelle, comme l'action de se marier ou de pacifier un conflit (p. 153). Mais sans doute le lecteur aurait-il eu besoin de juger, sur quelques exemples, que ces actions essentiellement symboliques et minimalement corporelles pouvaient vraiment être expliquées par le modèle volitionniste élaboré sur l'exemple d'actions corporelles simples. Mais laissons ce point de côté et considérons comment la théorie volitionniste peut rendre compte de l'action simple de Pierre.

Nous l'avons dit, si Pierre lève son bras pour attraper une pomme, c'est en vertu d'une volition correspondante. L'action dont Pierre est l'agent, l'action d'attraper une pomme, est causée, entretenue et guidée (p. 159) par une volition qui a la pomme pour cible. Une volition est un événement, pas une action. Si c'est Pierre qui lève le bras pour se saisir de la pomme, ce n'est pas Pierre qui fait qu'il a la volition correspondante. La volition advient et l'organisme dans lequel elle advient, dans notre cas Pierre, se met à agir, à produire des changements dans le monde. D'où sort cette volition? Pour simplifier: a) de la rétention d'expériences plaisantes faites, par le passé, à l'occasion de la dégustation involontaire de pommes; b) de la petite faim que Pierre éprouve présentement ; c) du surgissement, dans son champ visuel, d'une belle pomme rouge perçue comme accessible. Qui opère le rapprochement de ces trois facteurs? 
Ce n'est évidemment pas Pierre. C'est le «cerveau » (p. 159) de Pierre qui fait «basculer l'agent dans l'état d'exécution », c'est lui qui "constitue» Pierre en agent voulant attraper la pomme pour la manger. Comme l'écrit nettement J. Proust, «ce basculement, entièrement causé, et donc passivement opéré, est source du vouloir et du sentiment d'agir qui lui est associé » (p. 159-160).

Que faut-il mettre sous ces notions de vouloir et de sentiment d'agir? Essentiellement ceci: que l'événement mental d'origine cérébrale en quoi consiste la volition ne passe pas inaperçu. A la différence d'un robot, un organisme vivant qu'une volition met en action se sent agir. En particulier, il y a une différence qualitative entre le fait, pour un organisme, d'être habité par une volition et le fait d'être habité par un désir ou par une émotion. Les volitions se signalent par une «qualité dynamique » qui fait la différence entre l'expérience passive caractéristique d'avoir froid ou faim par exemple et l'expérience active d'être en train d'attraper une pomme. Un aspect de cette qualité dynamique est notamment constitué par le sentiment d'effort que J. Proust ne lie pas, comme Maine de Biran, à la «veille du Moi », mais à la copie d'efférence de la commande envoyée par le cerveau vers les muscles lorsque celui-ci fait basculer l'agent dans l'action (p. 192-193).

Telle est donc l'explication philosophique que J. Proust croit pouvoir donner de l'action simple de Pierre. Pierre est un organisme vivant qui se trouve être équipé pour avoir des volitions, c'est-à-dire pour être basculé par son cerveau dans l'action. Et comme tout organisme vivant, quel qu'en soit le degré de complexité, lorsque Pierre est basculé dans l'action, il vit, du dedans, certaines expériences caractéristiques. On ne doit pas dire (même s'il arrive parfois à J. Proust de le dire, par exemple p. 174) que Pierre perçoit ses volitions, car ce serait supposer que Pierre est en regard de ses volitions et reconduire ainsi le modèle de l'homoncule. Non, l'organisme vivant qu'est Pierre se trouve simplement mis en action de manière consciente, c'est-à-dire de telle sorte qu'en agissant il a des expériences caractéristiques et se rapporte représentativement à sa cible. En principe, Pierre pourrait être un zombie, car, comme l'écrit J. Proust p. 252, un robot peut avoir part à l'action volontaire. Mais le fait qu'il ait une conscience d'agir n'est pas un supplément gratuit à sa qualité objective d'agent. La conscience permet en effet de «favoriser le traitement ouvert et globalisant de l'information" (p. 179). Un organisme vivant se tire donc mieux d'affaire qu'un zombie. Il peut interrompre son action ou la relancer sur d'autres pistes parce qu'il est pathologiquement concerné par ce qu'il fait (p. 198).

A-t-on, à ce point, suffisamment équipé Pierre en processus cérébraux et mentaux pour que celui-ci puisse nous parler de son action? Si on demandait à Pierre: "Que fais-tu ? ", pourrait-il nous répondre : "J'attrape une pomme » ? La réponse est négative. L'organisme vivant que nous venons de décrire est un agent multi-tâches. Au gré des volitions qui se produisent en lui, il peut exécuter de manière consciente des actions différentes. Mais s'il sait ce qu'il fait, il ne se sait pas le faire. Il est tout à l'exécution de son action, à la cible 
de son action, à la distance à laquelle cette cible se trouve encore, etc., mais il n'est pas équipé pour former une pensée ayant pour objet l'action elle-même en tant que c'est lui qui l'accomplit. L'agent qu'on a décrit a beaucoup de ressemblances avec celui dépeint par Hobbes au chapitre VI du Léviathan consacré aux «commencements intérieurs des mouvements volontaires ». Il est un agent conscient, mais pas un agent conscient de soi. S'il pouvait parler de son action, il parlerait de ce qu'il fait, mais pas du fait que c'est lui qui le fait. Il emploierait un langage impersonnel, il dirait, par exemple, "Il y a attrapage de pomme ", mais il ne serait pas question de lui.

Cet agent hobbésien n'est donc pas encore suffisamment équipé pour pouvoir parler de ce qu'il fait, pour en porter la responsabilité aux yeux des autres, pour en rendre en raison ou s'en mordre les doigts. Si cet agent est bien un aspect de Pierre, c'est au mieux la bête qui est dans Pierre, ce que Pierre a gardé en lui des origines de son espèce. Mais si Pierre peut nous parler de ce qu'il fait, c'est qu'il possède, en sus de l'équipement de tout agent multitâches, un équipement neurologique et cognitif supplémentaire qui fait de Pierre une personne.

Quel est cet équipement supplémentaire? Il consiste, en premier lieu, dans la capacité à exécuter des actions mentales. Une action mentale est une action ayant pour cible des états mentaux. Soit par exemple l'état mental consistant à percevoir une pomme rouge. Une action mentale ayant pour cible cet état mental sera par exemple l'action de rendre claire et distincte cette perception, en se rendant attentif à l'objet rouge. J. Proust indique, p. 272, qu'une action mentale, étant une action volontaire, "implique une volition ». Si J. Proust ne décrit pas en détail comment ces volitions de second ordre se trouvent produites, on peut conjecturer que le mécanisme doit lui aussi faire appel à la mémoire. Le cerveau de l'agent aura gardé la trace d'épisodes antérieurs où, de manière involontaire, des états mentaux auront été modifiés de manière endogène jusqu'à atteindre un résultat dont un profit aura résulté. Le retour de circonstances semblables pourra donc entraîner que le cerveau de l'agent «fasse basculer» celui-ci dans l'action mentale. Une volition de mieux percevoir surgira dans l'agent et il fera ce qu'il faut pour l'exécuter, par exemple en écarquillant les yeux.

Un agent capable, non seulement d'actions corporelles, mais aussi d'actions mentales, est-il, de ce fait même, un agent capable de porter en première personne la responsabilité de ses actions ? La réponse est de nouveau négative. De même qu'un agent corporel multi-tâches accomplit successivement des actions différentes de façon à coïncider chaque fois avec l'action nouvelle qu'il exécute, un «agent mental » ne sera qu'un agent multi-tâches plus élaboré. Si par exemple il exécute l'action d'attraper une pomme, il pourra aussi accomplir l'action double de bien attraper cette pomme ou de l'attraper discrètement. Sa conscience d'agir sera donc plus riche : elle enveloppera non seulement l'ensemble des repères de l'action (la cible principale, les cibles instrumentales, etc.), mais aussi la modalité d'exécution de l'action. Si on lui prête un langage, 
il continuera lui aussi à s'exprimer de façon impersonnelle, mais, à la différence de l'agent hobbésien précédent, il agrémentera ses énoncés pratiques impersonnels de divers adverbes. Sa conscience d'agir modifiée ne se contentera pas d'un «Il y a attrapage de pomme ", mais pourra aller jusqu'à «Il y a un difficile attrapage de pomme» ou "il y a un discret attrapage de pomme».

Comment dès lors faire parler notre agent de lui-même ? Comment lui faire dire " je » ? Si l'on suit J. Proust, il faut lui prêter une capacité supplémentaire, une capacité en plus du pouvoir d'accomplir des actions mentales et que l'on peut décrire comme la capacité d'apercevoir génériquement ce que l'on fait quand on accomplit une action mentale. D'après J. Proust qui reprend ici une expression de Kant (CRpure, B 67) ayant eu une belle postérité chez Fichte et chez Maine de Biran, quand un agent accomplit une action mentale, il "s'auto-affecte». Mieux percevoir, mieux connaître, croire moins hasardeusement, etc., c'est à chaque fois, pour l'agent qui agit ainsi mentalement, non seulement "se manipuler soi-même" (p. 273), mais aussi s'autoaffecter. La thèse centrale de J. Proust est alors que pour qu'un agent puisse dire " je ", pour qu'il puisse non seulement parler de son actuel attrapage de pomme, mais se poser comme l'auteur de cette action, il faut et il suffit que l'agent ait «la conscience de pouvoir s'auto-affecter » c'est-à-dire « le souvenir de s'être auto-affecté joint à la conscience d'être en mesure, maintenant, de le faire » (p. 274). Concrètement, cela signifie que si un agent peut dire: «J'attrape une pomme ", plutôt que : "Il y a attrapage de pomme » ou : «Il y a difficile attrapage de pomme ", c'est parce qu'il a non seulement conscience de l'action qu'il exécute, voire de la modalité de son exécution, mais qu'il a en outre conscience de pouvoir modifier du dedans cette action, soit en la réorientant, soit en l'interrompant, soit en la défendant argumentativement aux yeux des autres. Pour parler de manière imagée, si l'agent «aperçoit » non seulement l'action, mais aussi lui-même en tant qu'accomplissant cette action, c'est parce qu'à la faveur de l'action qu'il accomplit et sur la base de sa mémoire d'actions mentales antérieures, il "aperçoit » le pouvoir générique qu'il possède sur cette action. Autrement dit encore: quand j'agis, si je me pose comme auteur de mon action, c'est parce que je me sais pouvoir modifier cette action ou modifier la modalité de son exécution.

Joëlle Proust affirme, p. 294, que sa caractérisation de la personnalité ou de la capacité des agents humains à se poser comme auteurs de leurs actions n'est pas «circulaire». D'après elle, la conscience de pouvoir s'auto-affecter n'est pas, à l'instar de ce que pensaient Fichte ou Maine de Biran, un pouvoir spirituel irréductible, le pouvoir d'auto-affection du Moi, mais c'est une capacité dont J. Proust dit qu'elle "résulte du processus méta-cognitif », qu'elle est « le produit de la méta-cognition » (p. 294). Examinons, pour finir, si c'est vraiment le cas. J. Proust insiste, en conclusion, sur le fait que «ce n'est pas l'agent qui forme ses volitions" (p. 307). Cette thèse nous paraît semblable à celle de Hobbes dans le texte précité du Léviathan et elle constitue, sans aucune doute, la seule façon d'immuniser le volitionnisme contre le risque de 
la régression ou de l'homonculisation. Mais que peut bien signifier le fait que l'agent, non pas ait conscience de s'auto-affecter, mais ait conscience de pouvoir s'auto-affecter? Notons qu'il est essentiel, pour J. Proust, de parler non pas d'une conscience de s'auto-affecter, mais d'une conscience de pouvoir le faire: car il s'agit de créditer l'agent humain, non pas d'une subjectivité épisodique, mais d'une personnalité transtemporelle. Il faut donc que l'agent puisse se détacher des actions qu'il se trouve en train d'exécuter, pour se distinguer d'elles et se poser comme l'auteur d'une lignée d'actions (puisque c'est bien là ce que nous sommes capables de faire). Or, répétons notre question: que peut bien signifier "avoir conscience de pouvoir s'auto-affecter » ? Ce n'est pas être conscient d'être actuellement en train de s'auto-affecter puisque même si l'agent exécute une action de premier ordre sans y mêler une action mentale de second ordre (par exemple s'il fait quelque chose machinalement), il peut se poser comme auteur de cette action de premier ordre. Or il serait par ailleurs curieux de soutenir que "avoir conscience de pouvoir s'auto-affecter » signifie «avoir conscience de pouvoir basculer dans l'autoaffection ", "avoir conscience de pouvoir se trouver dans la situation d'accomplir une action de second ordre ». On jouerait ici clairement sur le mot «pouvoir» qu'on prendrait tantôt, pour employer le vocabulaire d'Aristote, pour une puissance active (la puissance de s'auto-affecter), tantôt pour une puissance passive (la puissance d'être mis en auto-affection). Il suit dès lors qu'à moins d'accepter cette interprétation curieuse, "avoir le pouvoir de s'autoaffecter » ne peut rien signifier d'autre qu'avoir conscience d'être en mesure de le faire à volonté.

Mais alors de deux choses l'une: ou bien l'agent a vraiment ce pouvoir, et dans ce cas, il peut se mettre lui-même en action sans avoir besoin d'y être basculé par l'occurrence d'une volition. Dans un schéma globalement hobbésien, on est donc amené à glisser un peu de biranisme pour donner à l'agent qu'on affirme par ailleurs constitué par ses volitions, le pouvoir de dire «je» et de se poser comme auteur de ses actions; ou bien l'agent a seulement l'illusion de pouvoir se mettre en action, il a tiré du souvenir de ses actions mentales antérieures la croyance fausse en un pouvoir générique d'auto-affection, mais la réalité est que, soit que ses actions soit de premier ordre, soit qu'elles soit mentales, il doit attendre d'être basculé dans l'action par son «cerveau » pour agir.

La lecture de la p. 304 de l'ouvrage de J. Proust où celle-ci mentionne la formule de Théodule Ribot selon qui " "Je veux" constate une situation » et où on peut également lire que «l'agent reçoit ses propriétés actives ", qu' « il ne les institue pas, qu'il «n'est pas en amont de l'action, parce qu'il ne domine pas ses propres états mentaux » accrédite l'interprétation déconstructionniste. La conviction d'être auteur de ses actions serait une illusion qui flotterait à la surface d'une réalité naturelle impersonnelle, un flatus vocis sans force causale. Mais, par ailleurs, tout au long de son chapitre VII, J. Proust semble prendre au sérieux la notion de personne et elle affirme qu' « être une personne 
n'est pas en son principe une fiction" (p. 291). Mais si ce qui est réel, c'est ce qui possède un rôle causal, alors la personne, conçue comme conscience permanente de pouvoir s'auto-affecter, c'est le rôle causal d'une puissance active qui semble fatalement reconduire le volitionnisme vers un homonculisme biranien.

Nous nous doutons bien que J. Proust ne se laissera pas enfermer dans les termes de cette antinomie. Mais, pour notre part, faute d'en connaitre encore la solution, nous conclurons par provision que la meilleure manière d'exorciser le biranisme, sans priver les personnes de toute espèce de réalité, c'est peut-être de renoncer au hobbisme. 